\title{
A mixed-effects expectancy-valence model for the Iowa gambling task
}

\author{
Chung-Ping Cheng \\ National Chengchi University, Taipei, Taiwan \\ Ching-Fan SheU \\ National Cheng Kung University, Tainan City, Taiwan \\ AND \\ NAI-SHING YeN \\ National Chengchi University, Taipei, Taiwan
}

\begin{abstract}
The Iowa gambling task (IGT; Bechara, Damasio, Damasio, \& Anderson, 1994) was developed to simulate real-life decision making under uncertainty. The task has been widely used to examine possible neurocognitive deficits in normal and clinical populations. Busemeyer and Stout (2002) proposed the expectancy-valence (EV) model to explicitly account for individual participants' repeated choices in the IGT. Parameters of the EV model presumably measure different psychological processes that underlie performance on the task, and their values may be used to differentiate individuals across different populations. In the present article, the EV model is extended to include both fixed effects and subject-specific random effects. The mixed-effects EV model fits the nested structure of observations in the IGT naturally and provides a unified procedure for parameter estimation and comparisons among groups of populations. We illustrate the utility of the mixed-effects approach with an analysis of gender differences using a real data set. A simulation study was conducted to verify the advantages of this approach.
\end{abstract}

The expectancy-valence (EV) model is a cognitive decision model developed by Busemeyer and Stout (2002) to decompose performance on the Iowa gambling task (IGT; Bechara, Damasio, Damasio, \& Anderson, 1994). The task is designed to simulate real-life decision making in a complex situation, and performance on the task indicates a person's ability to anticipate negative consequences of disadvantageous choices.

Typically, the analysis of data in IGT using the EV model is conducted in two stages. First, separate parameter estimates are obtained from fitting the model to the repeated choices of each individual in a group. Second, standard statistical procedures such as analysis of variance may then be used to compare group differences using the estimated values of model parameters as raw data. The two-stage approach, however, can be biased and inefficient — biased because information contained in the standard errors of parameter estimates is ignored in the second stage, and inefficient because no mechanism exists in the analysis to account for the tendency of individuals in the same group to behave similarly. The two-stage analysis also lacks ways to directly link experimental manipulations to group comparisons.

The drawbacks of a two-stage analysis of the IGT data may be nullified by a mixed-effects modeling approach. Here, we propose a mixed-effects EV model by incorpo- rating both subject-specific random effect parameters and group-specific fixed-effect parameters so that parameter estimation and group comparison can be achieved within a single framework. By pooling information from groups of individuals, the mixed-effects EV model may attain superior statistical power for detecting group differences, when such differences exist.

In this article, we first briefly review the IGT and the EV model. We then describe the mixed-effects EV model and show how to implement parameter estimation using SAS PROC NLMIXED (Wolfinger, 1999). In the third section we compare the proposed mixed-effects approach with the two-stage procedure by analyzing a real data set for gender differences in the IGT. The fourth section reports a simulation study to validate the superior power of the proposed approach, and, finally, we discuss the advantages of the approach and the use of mixed (or hierarchical) models in analyzing cognitive performance.

\section{The IGT and EV Model}

The IGT is a card-selection task with four decks of cards that vary in gains and losses. At the outset, participants are given a certain amount of hypothetical money to play the game. The goal is to maximize one's profit at the end of the game (usually, after 100 trials). At each trial, the 
participant is required to choose one card out of the four decks. Feedback about gains and losses is provided after a choice is made. Two decks of cards are associated with a large constant gain but a negative expected gain in the long run; the other two decks of cards are associated with a smaller constant gain but a positive expected gain in the long run. Poor performance in the task indicates a failure to anticipate negative consequences of bad choices. Compared with normal individuals, patients with damage to prefrontal cortex selected cards in the IGT in ways that showed insensitivity to future consequences (Bechara et al., 1994). The task has been widely used to characterize decision-making deficits in different populations, such as individuals with addiction problems (Bechara \& Damasio, 2002; Verdejo-Garcia et al., 2007), aging populations (Isella et al., 2008), and women with bulimia nervosa (Boeka \& Lokken, 2006).

The EV model, proposed by Busemeyer and Stout (2002), is designed to measure the different psychological processes that underlie performance on the IGT. The model assumes that the decision maker at any given trial integrates gains and losses of all previous trials to form an expectancy valence for the present trial on which the probability of the next trial depends. More specifically, the valence of the payoffs on a trial is a weighted average of the gains and losses. The expectancy of a deck at a given trial is the expectancy of the deck at a previous trial plus the discrepancy between the valence of the current choice and the expectancy of the deck at a previous trial adjusted by learning. Finally, the choice probability for a particular deck is determined by the strength of that deck relative to the sum of the strengths of all decks. In other words, the underlying decision-making processes in the IGT identified by the EV model are motivational, learning, and response processes. Given an individual participant's choice data, Busemeyer and Stout estimated parameters for each of these three processes via the maximum likelihood method.

Estimated values of these model parameters have been used to compare healthy young adults against other target groups. Yechiam, Busemeyer, Stout, and Bechara (2005) plotted 10 different populations of participants according to their performance on the IGT in reference to a control group, with their locations on the map determined by the differences in estimated values of the learning-rate parameter (the vertical axis) against the differences in estimated values of the motivation parameter (the horizontal axis). The standard errors of the differences of estimates are shown as a cross centered for each population. Huntington patients, for instance, are located in the upper right quadrant of the map, reflecting a greater sensitivity to gains (relative to losses) and more attention to the most recent outcomes as opposed to past outcomes, compared with the control group.

Comparisons of model parameter estimates on such a map reveal differences in decision-making processes among distinct groups. Thus, it is important to be able to accurately and efficiently estimate parameters of the EV model from IGT data. For any given population of par- ticipants, a set of three parameter estimates was obtained from each individual (Yechiam et al., 2005). These authors summarized the distribution of parameter estimates for each population by computing the average and standard deviation for each parameter. To compare two distinct groups of populations, the differences between parameter estimates were computed. A major drawback of this approach is that it ignores the multilevel or hierarchical structure of the IGT data. Clearly, there exist trial-by-trial variations within the same individual, subject-by-subject variations within the same group of individuals, and, finally, between-group differences. Participants in the IGT are random effects because the scope of inference pertains not only to the effects present in those who participated in the study, but also to the effects present in the entire population. On the other hand, the distinct groups of populations are fixed effects. These considerations lead us to propose a mixed-effects EV model that incorporates both subject-specific random-effect parameters and groupspecific fixed-effect parameters, so that parameter estimation and group comparison can be achieved within a single statistical framework. By pooling information from groups of individuals, the mixed-effects EV model gains statistical power for detecting group differences, when such differences exist.

\section{The Mixed-Effects EV Model}

The mixed-effects EV model is specified as follows:

$$
\begin{aligned}
& v_{t}=w_{i} * G_{t}+\left(1-w_{i}\right) * L_{t} . \\
& E v_{t, k}=\left(1-a_{i}\right) E v_{t-1, k}+a_{i} * v_{t} \quad \text { if } D_{t, k}=1 \text {, } \\
& =E v_{t, k} \\
& p_{t+1, k}=\frac{\exp \left[E v_{t, k} *(t / 10)^{c_{i}}\right]}{\sum_{j=1}^{4} \exp \left[E v_{t, j} *(t / 10)^{c_{i}}\right]},
\end{aligned}
$$

where $v_{t}$ is the valence at trial $t . G_{t}$ and $L_{t}$ indicate gain and loss at trial $t . E v_{t, k}$ denotes the EV of choosing deck $k$ at trial $t$. The indicator variable $D_{t, k}$ is 1 if at trial $t$ the deck $k$ is chosen; otherwise, $D_{t, k}=0 . p_{t, k}$ denotes the probability of choosing deck $k$ at trial $t$.

Equations 1, 2, and 3 are identical to the original formulation of the EV model (Busemeyer \& Stout, 2002) except for $w$, which is the motivation parameter, indicating a decision maker's attention to gain relative to loss, $a$, which represents the rate at which EV is updated, and $c$, which specifies the changes in the sensitivity of the choice probability to expectancy. These are assumed to be subject-specific random effects and are indexed by $i$, for the $i$ th participant.

In fitting the EV model to IGT data, our experience showed that the estimation cost a lot of time, and that estimates obtained from running the optimization routine were very susceptible to the starting values of the parameters. This might be because the third parameter $c$ in the original formulation of the EV model is the exponent of a power function, which, in turn, is itself exponentiated. 
Tiny changes in the magnitude of $c_{i}$ can yield wildly different predicted choice probabilities. To overcome such problems, we reparameterize Equation 3 into Equation 4 with $c_{i}^{\prime}=10^{c_{i}}$.

$$
p_{t+1, k}=\frac{\exp \left(E v_{t, k} * c_{i}^{\prime \log _{10} t-1}\right)}{\sum_{j=1}^{4} \exp \left(E v_{t, j} * c_{i}^{\prime \log _{10} t-1}\right)} .
$$

We further assume that the random-effect parameters are sampled from independent and identically distributed multivariate normal distributions as shown below in Equation 5, where $X$ is a design matrix with fixed covariates. For example, with only two distinct groups of participants, the group membership is dummy coded by an indicator $X$, resulting in the between-group difference being represented through model parameters $w_{1}, a_{1}$, and $c_{1}^{\prime}$. Variances of parameters of $w_{i}, a_{i}$, and $c_{1}^{\prime}$ are indicated by $\sigma_{w w}, \sigma_{a a}$, and $\sigma_{c^{\prime} c^{\prime}}$, respectively. Correlations between pairs of parameters are indicated by their corresponding subscripts.

\section{Parameter Estimation of the EV Model}

Busemeyer and Stout (2002) estimated parameters of the EV model by the maximum likelihood method with Nelder-Mead simplex optimization (O'Neill, 1971). Under reparameterization of the sensitivity parameter in the EV model, three methods we have investigatedsimplex, conjugate-gradient, and double dogleg optimization (SAS Institute, 2004) - all exhibited better converging performance under the reparameterization. The latter two methods appeared to be computationally faster than the simplex routine.

We note that since Equation 2 is recursively defined, the EV model can be reformulated as a single equation, presented below (Equation 6).

The probability of choosing deck $k$ at trial $t+1$ is clearly a nonlinear function of cumulative gains and losses, as well as the responses of previous trials. In other words, the EV model is a nonlinear regression model. Thus, fitting the EV model to IGT data can readily be implemented with built-in routines, such as PROC NLIN (SAS Institute, 2004) or nls in R (R Development Core Team, 2006).

\section{Parameter Estimation of the \\ Mixed-Effects EV Model}

The extended EV model with subject-specific random effects that we proposed is recognized as a nonlinear mixed-effects model (see, e.g., Pinheiro \& Bates, 1995). Either the nlme package in R (Pinheiro \& Bates, 2000) or the NLMIXED procedure (SAS Institute, 2004) can be used to estimate parameters of nonlinear mixed models. For this study, parameters of the mixed-effects EV model were estimated using SAS following Sheu, Chen, $\mathrm{Su}$, and Wang's (2005) discussion on the implementation of nonlinear mixed-effects models in PROC NLMIXED.

The Appendix presents a code example of the implementation. To start with, the DATA step reads a properly arranged data set from an IGT experiment. Each row in the data set represents a participant's trial. Every row consists of the participant's ID, trial, response, number of cards chosen at each deck before this trial, and a covariate. The responses are regressed onto payoffs of previous trials. The sequences of values of the gains and losses of each deck before each trial should also be listed. Because each deck consists of 40 cards in an ordinary IGT, the length of sequence representing values of losses of each deck at previous trials is set to 40 , and the unused columns should be filled with zeroes. Given a deck in the IGT, the value of gain is constant; only a single variable is required for the gain of previous trials.

PROC NLMIXED evokes the estimation procedure of parameters of the mixed-effects model. We suggest using the maximum likelihood procedure with conjugategradient optimization and importance sampling (SAS Institute, 2004). The suggested procedure can be implemented by setting TECH = congra and METHOD = isamp, respectively. TECH selects the optimization routine for maximizing the log-likelihood function, and METHOD specifies the method for approximating the integral of the likelihood over the random effects. QPOINTS determines the number of quadrature points in the approximation of the integral. ARRAY reads from a previous SAS data set or creates an array to store variables in the mixed-effects EV model, to simplify the code. PARMS sets the starting value of parameters of the model. The following part of the code calculates every trial's valence and EV and then

$$
\begin{aligned}
& \left(w_{i}, a_{i}, c_{i}^{\prime}\right) \sim N\left[w_{0}+w_{1} X, a_{0}+a_{1} X, c_{0}^{\prime}+c_{1}^{\prime} X,\left(\begin{array}{ccc}
\sigma_{w w} & \\
r_{a w} \sqrt{\sigma_{a a} \sigma_{w w}} & \sigma_{a a} & \\
r_{c^{\prime} w} \sqrt{\sigma_{c^{\prime} c^{\prime}} \sigma_{w w}} & r_{c^{\prime} a} \sqrt{\sigma_{c^{\prime} c^{\prime}} \sigma_{a a}} & \sigma_{c^{\prime} c^{\prime}}
\end{array}\right)\right] \\
& p_{t+1, k}=\frac{\exp \left\{\sum_{l=1}^{t} D_{l, k} * a_{i} *\left(1-a_{i}\right)^{\sum_{m=1}^{t} D_{m, k}-1-\sum_{m=1}^{l} D_{m, k}} *\left[w_{i} * G_{t}+\left(1-w_{i}\right) * L_{t}\right] * c_{i}^{\prime \log _{10} l-1}\right\}}{\sum_{j=1}^{4} \exp \left\{\sum_{l=1}^{t} D_{l, j} * a_{i} *\left(1-a_{i}\right)^{\sum_{m=1}^{t} D_{m, j}-1-\sum_{m=1}^{l} D_{m, j}} *\left[w_{i} * G_{t}+\left(1-w_{i}\right) * L_{t}\right] * c_{i}^{\prime \log _{10} l-1}\right\}} \\
& =f\left(G_{1}, G_{2}, \ldots, G_{t}, L_{1}, L_{2}, \ldots, L_{t}, D_{1, k}, D_{2, k}, \ldots, D_{t, k} \mid a_{i}, w_{i}, c_{i}^{\prime}\right) .
\end{aligned}
$$


forms the choice probabilities of four decks according to the reformulation of the EV model (i.e., Equation 6). MODEL associates the dependent variable "response" with its probability function setting by the subcommand GENERAL with log-probability, which will sum up to the log-likelihood function, in the bracket. RANDOM specifies the random effects as a multivariate normal distribution. SUBJECT identifies that random effects vary across subjects. The code can also be used to estimate the parameters of the original EV model by leaving out the random effects and modifying the specification of parameters.

\section{Gender Differences in IGT}

The data from a sample of 13 male and 15 female Taiwanese college students performing the IGT were analyzed using the original EV model in a two-stage analysis and using the mixed-effects version. (The data came from the third author's larger study on the somatic marker hypothesis.) The ability of the two models to detect gender differences in IGT performance was the focus of comparison. To check the feasibility of the EV model in our IGT data, we calculated differences in 2 log-likelihood of the original EV model contrasted to baseline multinomial model (Busemeyer \& Stout, 2002). The positive difference indicates that the EV model performs better than the baseline model in the data. All participants' differences in 2 log-likelihood were positive. We also checked the feasibility of the mixed-effects EV model by calculating the difference in $2 \log$-likelihood of the mixed-effects EV model in reference to the original EV model. The difference in $2 \log$-likelihood between the two model fits to the present data is 293.7, with the original EV model having 72 more parameters. The ratio of $2 \log$-likelihood to the number of extra parameters is smaller than 5 , indicating that the two model fits are comparable (Marsh \& Hocevar, 1985).

Parameter estimates and standard errors obtained from the two approaches are shown in Table 1. The estimated parameter values are not much different except for the response-sensitivity parameter. The standard errors of the motivation parameter and learning rate parameter for the mixed-effects EV model are only about half of those for the original EV model. Large variation and uncertainty in single-subject estimates were also observed by Wetzels, Vandekerckhove, Tuerlinckx, and Wagenmakers (2008).

We also calculate the $95 \%$ confidence intervals for mean differences across gender based on the two approaches. No gender differences were found among the resulting pa- rameter estimates obtained using the two-stage analysisthat is, fitting the original $\mathrm{EV}$ model to individual data one at a time and following a two-independent-samples $t$ test. In contrast, results of the mixed-effects analysis, shown on the right of Table 1, made clear that there are statistically significant gender differences for the motivation parameter between the sexes. Results based on the mixed-effects EV model suggested that male college students paid more attention to gain compared with their female counterparts. But there were no significant gender differences in learning or response sensitivity.

Bolla, Eldreth, Matochik, and Cadet (2004) found that male adults performed better than female adults in the IGT, and that only male adults showed a significant learning effect. In rodents, van den Bos, Lasthuis, den Heijer, van der Harst, and Spruijt (2006) also found a learning effect in favor of male mice. However, both Bolla et al. and van den Bos et al. used the net score (the number of good decks chosen minus the number of bad decks chosen) in their statistical analyses without evoking the EV model. Therefore, their results and ours cannot be directly compared. Further research is needed to clarify the gender differences in the IGT.

A significant correlation $(r=-.31, p<.05)$ between the motivation parameter and the response-sensitivity parameter was observed from fitting the mixed-effects EV model to data. Although the original EV model does not specify correlations among model parameters, we found similar patterns of correlations among model parameters by calculating the Pearson correlation coefficients based on estimated values of individuals' parameters of the EV model. The value of the correlation coefficient between estimates of the motivation and response-sensitivity parameters was $-.63(p<.05)$.

\section{Simulation}

A simulation study was conducted to directly verify the advantage of the analysis by the mixed-effects EV model over the two-stage analysis using the original EV model to detect group differences. We simulated IGT data from an experiment with two groups (a control group and a treatment group). In the first group $(n=21)$, means and standard deviations, respectively, of three parameters, $w, a$, and $c^{\prime}$, were set at .70 and .10;.007 and .002; and .40 and .10 . In the second group $(n=21)$, the means and standard deviations of those three parameters were set at .78 and $.10 ; .0086$ and .002 ; and .48 and .10 . The standardized differences of $w_{i}, a_{i}$, and $c_{i}^{\prime}$ between the two groups

Table 1

Means, Standard Deviations, and 95\% Confidence Intervals for Mean Differences of Estimates of Parameters of the Expectancy-Valence (EV) Model by Different Approaches Between Female $(n=13)$ and Male $(n=15)$ College Students

\begin{tabular}{|c|c|c|c|c|c|c|c|c|c|c|}
\hline & \multicolumn{5}{|c|}{ Two-Stage Analysis } & \multicolumn{5}{|c|}{ Mixed-Effects EV Model } \\
\hline & \multicolumn{2}{|c|}{ Female } & \multicolumn{2}{|c|}{ Male } & \multirow{2}{*}{$\begin{array}{c}95 \% \mathrm{CI} \\
\text { for Mean } \\
\text { Difference }\end{array}$} & \multicolumn{2}{|c|}{ Female } & \multicolumn{2}{|c|}{ Male } & \multirow{2}{*}{$\begin{array}{c}95 \% \text { CI } \\
\text { for Mean } \\
\text { Difference }\end{array}$} \\
\hline & $M$ & $S D$ & $M$ & $S D$ & & $M$ & $S D$ & $M$ & $S D$ & \\
\hline$w$ & .820 & .174 & .855 & .147 & $-.091, .162$ & .740 & .074 & .797 & .074 & $.016, .099$ \\
\hline$a$ & .009 & .005 & .009 & .008 & $-.005, .005$ & .009 & .002 & .007 & .002 & $-.004, .006$ \\
\hline$c^{\prime}$ & .262 & .350 & .182 & .263 & $-.324, .163$ & .033 & .236 & .028 & .236 & $-.068, .058$ \\
\hline
\end{tabular}


were set at .8 , a conventional criterion of large effect size (Cohen, 1988). We set the sample sizes of both groups to 21 , which resulted in power equal to .8 if an ordinary independent $t$ test is conducted. The group differences of the three parameters in simulated data were analyzed by two approaches. Percentages of significant results (i.e., power) of $w_{i}, a_{i}$, and $c_{i}^{\prime}$ in 100 replications using the twostage analysis were $.39, .23$, and .01 , respectively. When the mixed-effects EV model was applied to the same simulated data, proportions of runs finding significant differences of $w_{i}, a_{i}$, and $c_{i}^{\prime}$ were increased to $.66, .40$, and .28 , respectively. These results suggested that the mixedeffects EV model could outperform the traditional twostage analysis in detecting true group differences.

\section{Discussion}

The aim of this article was to extend the EV model to include both fixed and random effects and to show that parameters of the mixed-effects version of the EV model can be efficiently estimated using general-purpose statistical software, such as SAS. Results from the analysis of a small real data set and a simulation study suggested that the extended model was more sensitive at detecting group differences by efficiently pooling information from all individuals. Since parameter estimates of the EV models from IGT data have been used to characterize differences in the decision-making processes among many clinical and nonclinical populations, the proposed mixed-effects EV model can potentially unify diverse empirical findings within a single statistical framework. Extending the EV model to include subject-specific random effects allows important model parameters to be simultaneously estimated for a population.

Although the demonstration presented in this study focuses on testing for group differences in IGT, other aspects of the decision-making processes in IGT can also be investigated using the mixed-effects EV model. For instance, with some minor modifications, the extended model can be applied to account for the relationship between decision-making ability and other continuous covariates, such as personality traits (Davis, Patte, Tweed, $\&$ Curtis, 2007) or age and IQ (Stout, Busemeyer, Lin, Grant, \& Bonson, 2004).

The two-stage analysis strategy is often employed in behavioral data analysis. Although a model may describe cognitive processes within an individual, data analysis using the model typically involves more than one individual. The practice of first estimating parameters by fitting the model to each individual and then comparing individual parameter estimates across different groups or conditions has intuitive appeal. Recent advances in multilevel modeling have shown that, despite its intuitive appeal, the twostage analysis suffers from many drawbacks, which can be eliminated by mixed-effects models. However, there are two distinctly different approaches to applying the mixed-effects methodology to account for variations between and within subjects. One approach directly applies the mixed-effects statistical methodology in the analysis of experimental data. For example, Hoffman and Rovine (2007) introduced the multilevel models to experimental psychologists by examples. Stockard, O'Brien, and Peters (2007) analyzed a modified IGT and a prisoner's dilemma game using mixed models without explicitly involving the EV model or any other process models.

Another approach to applying mixed-effects modeling involves the generalization of the original quantitative model of a cognitive task to include both fixed and random effects. The proposed mixed-effects EV model adds another case study in the latter category. For example, the Deese/ Roediger-McDermott false memory paradigm (Roediger \& McDermott, 1995) is usually analyzed in the framework of signal detection theory. Sheu, Lee, and Shih (2008) suggested accounting for participants' variability by introducing random effects into the signal detection model.

The distinction between the two mixed modeling approaches might be blurred, however, in a Bayesian framework (Wetzels et al., 2008). In a hierarchical approach to modeling response time distributions, Rouder, $\mathrm{Lu}$, Speckman, Sun, and Jiang (2005) demonstrated that their proposed mixed-effects model was better at capturing the effects of experimental manipulations than standard methods of analyzing reaction time in individual or aggregate-level data. Since experiments involving multiple trials by a sample of individuals contain both withinand between-subjects variations, the proposed mixed modeling approach may be preferable to the two-stage approach and should receive serious consideration from experimental psychologists in their data analyses.

\section{AUTHOR NOTE}

The authors thank an anonymous reviewer, whose comments we found helpful in our revision of the article. The work here was supported by a grant to the first author from the National Science Council of Taiwan (NSC 97-2410-H-004-146). The second author is supported, in part, by a grant from the same funding agency (NSC 96-2413-H-006-003). Correspondence concerning this article should be addressed to C.-P. Cheng, Department of Psychology, National Chengchi University, No. 64, Sec. 2, Zhi-nan Rd., Taipei, Taiwan (e-mail: cpcheng@nccu.edu.tw).

\section{REFERENCES}

Bechara, A., Damasio, A. R., Damasio, H., \& Anderson, S. W. (1994). Insensitivity to future consequences following damage to human prefrontal cortex. Cognition, 50, 7-15.

Bechara, A., \& Damasio, H. (2002). Decision-making and addiction (Part I): Impaired activation of somatic states in substance-dependent individuals when pondering decisions with negative future consequences. Neuropsychologia, 40, 1675-1689.

BoekA, A. G., \& LoKken, K. L. (2006). The Iowa gambling task as a measure of decision making for women with bulimia nervosa. Journal of the International Neuropsychological Society, 12, 741-745. doi:10.10170S1355617706060887

Bolla, K. I., Eldreth, D. A., Matochik, J. A., \& CAdet, J. L. (2004). Sex-related differences in a gambling task and its neural correlates. Cerebral Cortex, 14, 1226-1232. doi:10.1093/cercor/bhh083

Busemeyer, J. R., \& Stout, J. C. (2002). A contribution of cognitive decision models to clinical assessment: Decomposing performance on the Bechara gambling task. Psychological Assessment, 14, 252-262.

CoHen, J. (1988). Statistical power analysis for the behavioral sciences. Mahwah, NJ: Erlbaum.

Davis, C., Patte, K., Tweed, S., \& Curtis, C. (2007). Personality traits associated with decision-making deficits. Personality \& Individual Differences, 42, 279-290. doi:10.1016/j.paid.2006.07.006

Hoffman, L., \& Rovine, M. J. (2007). Multilevel models for experimental psychologists: Foundations and illustrative examples. Behavior Research Methods, 39, 101-117. 
Isella, V., Mapelli, C., Morieli, N., Pelati, O., Franceschi, M., \& Appollonio, I. M. (2008). Age-related qualitative and quantitative changes in decision making ability. Behavioral Neurology, 19, 59-63.

Marsh, H. W., \& Hocevar, D. (1985). Application of confirmatory factor analysis to the study of self-concept: First- and higher-order factor models and their invariance across groups. Psychological Bulletin, 97, 562-582.

O'NeILl, R. (1971). Algorithm AS 47: Function minimization using a simplex procedure. Applied Statistics, 20, 338-345.

Pinheiro, J. C., \& BATES, D. M. (1995). Approximations to the loglikelihood function in the nonlinear mixed-effects model. Journal of Computational \& Graphical Statistics, 4, 12-35.

Pinheiro, J. C., \& Bates, D. M. (2000). Mixed-effects model in S and S-Plus. New York: Springer.

R Development Core Team (2006). $R$ : A language and environment for statistical computing [Computer software and manual]. Vienna: $\mathrm{R}$ Foundation for Statistical Computing.

Roediger, H. L., III, \& McDermott, K. B. (1995). Creating false memories: Remembering words not presented in lists. Journal of Experimental Psychology: Learning, Memory, \& Cognition, 21, 803-814.

Rouder, J. N., Lu, J., Speckman, P. L., Sun, D., \& Jiang, Y. (2005). A hierarchical model for estimating response time distributions. Psychonomic Bulletin \& Review, 12, 195-223.

SAS InstituTE, InC. (2004). SAS/STAT user's guide (Version 9.1). Cary, NC: Author.

Sheu, C.-F., Chen, C.-T., Su, Y.-H., \& WAng, W.-C. (2005). Using SAS PROC NLMIXED to fit item response theory models. Behavior Research Methods, 37, 202-218.

Sheu, C.-F., LeE, Y.-H., \& SHIH, P.-Y. (2008). Analyzing recognition performance with sparse data. Behavior Research Methods, 40, 722 727.

Stockard, J., O'Brien, R. M., \& Peters, E. (2007). The use of mixed models in a modified Iowa gambling task and a prisoner's dilemma game. Judgment \& Decision Making, 2, 9-22.

Stout, J. C., Busemeyer, J. R., Lin, A., Grant, S. J., \& Bonson, K. R. (2004). Cognitive modeling analysis of the decision-making processes used by cocaine abusers. Psychonomic Bulletin \& Review, 11, 742747.

van den Bos, R., Lasthuis, W., Den Heijer, E., van der Harst, J., \& SPRuIJT, B. (2006). Toward a rodent model of the Iowa gambling task. Behavior Research Methods, 38, 470-478.

Verdejo-Garcia, A., Benbrook, A., Funderburk, F., David, P., Cadet, J. L., \& Bolla, K. I. (2007). The differential relationship between cocaine use and marijuana use on decision-making performance over repeat testing with the Iowa gambling task. Drug \& Alcohol Dependence, 90, 2-11. doi:10.1016/j.drugalcdep.2007.02.004

Wetzels, R., Vandekerckhove, J., Tuerlinckx, F., \& WagenmaKERS, E.-J. (2008, July). Bayesian parameter estimation in the expectancy valence model of the Iowa gambling task. Paper presented at the 41st Mathematical Psychology Meeting, Washington, DC.

Wolfinger, R. D. (1999). Fitting nonlinear mixed models with the new NLMIXED procedure. In Proceedings of the 24th Annual SAS Groups International Conference (SUGI24) (Paper 287). Gary, NC: SAS Institute.

Yechiam, E., Busemeyer, J. R., Stout, J. C., \& Bechara, A. (2005) Using cognitive models to map relations between neuropsychological disorders and human decision making deficits. Psychological Science, 16, 973-978. doi:10.1111/j.1467-9280.2005.01646.x 


\section{APPENDIX}

DATA igt;

INFILE 'igt.dat';

INPUT id trial response numdeck1- numdeck $4 \mathrm{x}$ gvalue1-gvalue 4

lvalue101-lvalue140 lvalue201-lvalue240

lvalue301-lvalue340 lvalue401-lvalue440;

PROC NLMIXED DATA=igt TECH=congra METHOD=isamp QPOINTS=25;

/*Store numbers of cards chosen at deck A to D before the present trial in an array.*/

ARRAY numdeck(4) numdeck1- numdeck4;

/*Store gain values of deck A to D in an array.*/

ARRAY gvalue(4) gvalue1-gvalue4;

/*Store loss values of cards chosen at deck A to D before the present trial in an array.*/

ARRAY lvalue(4,40) lvalue101-lvaluea140 lvalue201-lvalue240

lvalue301-lvalue340 lvalue401-lvalue440;

/*Create arrays for expectancy-valences and choice probabilities of four decks.*/

ARRAY ev(4); ARRAY p(4);

PARMS w $0=.5 \mathrm{a} 0=.01$ cprime $0=.5 \mathrm{w} 1=.0 \mathrm{a} 1=.0$ cprime $1=.0$ $\mathrm{sww}=.01 \mathrm{saa}=.001 \mathrm{scc}=.01$ corraw $=0$ corrcw $=0$ corrca $=0$;

saw $=$ corraw $^{*}$ sqrt $\left(\right.$ saa $^{*}$ sww $)$;

$\mathrm{scw}=$ corrcw* sqrt $^{\left(\mathrm{scc}^{*} \mathrm{sww}\right)}$;

sca $=$ corrca $^{*}$ sqrt $\left(\right.$ scc $^{*}$ saa $)$;

/*Calculate the expectancies and probabilities of four decks.*/

$\mathrm{w}=\left(\mathrm{w} 0+\mathrm{w} 1 *_{\mathrm{x}}+\mathrm{wr}\right)$

$\mathrm{a}=(\mathrm{a} 0+\mathrm{a} 1 * \mathrm{x}+\mathrm{ar})$;

cprime $=\left(\right.$ cprime $0+$ cprime $*^{*} \mathrm{x}+$ cprimer $)$;

DO $\mathrm{j}=1 \mathrm{TO} 4$;

$\mathrm{ev}[\mathrm{j}]=0$;

DO $\mathrm{i}=1$ TO numdeck $[\mathrm{j}]$

END;

ev $[\mathrm{j}]=\operatorname{ev}[\mathrm{j}]+((1-\mathrm{a}) * *($ numdeck $[\mathrm{j}]-\mathrm{i}) * \mathrm{a}) *\left(\mathrm{w}^{*}\right.$ gvalue $[\mathrm{j}]+(1-\mathrm{w}) * 1$ value $\left.[\mathrm{j}, \mathrm{i}]\right)$;

END;

DO $\mathrm{j}=1$ TO 4;

$\mathrm{p}[\mathrm{j}]=\exp \left(\operatorname{ev}[\mathrm{j}] *\right.$ cprime $^{* *}(\log 10($ trial $\left.)-1)\right)$;

if $($ numdeck $[j]=40)$ then $p[j]=0$;

END;

$\mathrm{p}=\mathrm{p} / \operatorname{sum}(\mathrm{p})$;

$\log \mathrm{P}=\log (\mathrm{p}[$ response $])$;

MODEL response $\sim$ GENERAL $(\log P)$;

RANDOM wr ar cprimer NORMAL([0,0,0],[sww,saw,saa,scw,sca,scc]) SUBJECT=id;

RUN; QUIT;

(Manuscript received November 7, 2008;

accepted for publication December 18, 2008.) 